\title{
Perú: aún estamos lejos de curarnos de la tuberculosis
}

\section{Peru: We are still far from a cure for tuberculosis}

Correspondencia Alberto Mendoza-Ticona mendozalberto@outlook.com

Recibido: 04/11/2018 Arbitrado por pares Aprobado: 14/11/2018

Citar como: MendozaTicona A. Perú: aún estamos lejos de curarnos de la tuberculosis. Acta Med Peru. 2018;35(4):195-6

\author{
Alberto Mendoza-Ticona ${ }^{1 \mathrm{a}, \mathrm{b}}$ \\ 1 Universidad Peruana Cayetano Heredia. Lima, Perú. \\ 2 Servicio de Infectología, Hospital de Emergencia Villa El Salvador. Lima, Perú. \\ a Médico Infectólogo, b Maestro en Epidemiología Clínica
}

El Perú se encuentra dentro de los 30 países con mayor carga de tuberculosis (TB) multidrogorresistente (TB MDR) en el mundo. En América, encabeza la lista de países con el mayor número de casos de TB MDR y TB extensamente resistente (TB XDR) y ocupa el tercer lugar entre los países con mayor número de casos de TB por cada 100 mil habitantes ${ }^{[1]}$.

Las intervenciones sanitarias implementadas en el país para afrontar esta situación aún son insuficientes. El Gobierno Nacional ha incrementado el presupuesto asignado al control de la TB y promulgado la Ley de Prevención y Control de la TB, que dispone la intangibilidad de este presupuesto [2]; sin embargo, las unidades ejecutoras del Ministerio de Salud (MINSA) y la Seguridad Social -como hospitales, regiones de salud e institutos- suelen incumplir esta Ley y disponen del presupuesto asignado para otros gastos.

El Perú, como miembro de la Organización de las Naciones Unidas, se comprometió en el año 2014 a desarrollar y cumplir las metas de la estrategia "Fin de la Tuberculosis", la cual propone eliminar la TB como problema de salud pública para el año 2035 [3]. Dicha estrategia establece que en los primeros cinco años, entre el 2016 y 2020, se deben implementar las intervenciones para el control de la TB que cuenten con evidencia sólida que las soporten. En los siguientes cinco años, deben implementarse métodos más eficaces de diagnóstico, nuevos regímenes de tratamiento y nuevas vacunas que disminuyan la incidencia y las fuentes de contagio; y en los últimos 10 años, entre el 2016 y 2035, se debe expandir el tratamiento preventivo, con regímenes cortos y efectivos.

Pero, como país, ¿cómo está el Perú en la meta de los primeros cinco años? Es decir, implementar lo que ya se sabe que funciona cerrando brechas existentes. A continuación, se presentan intervenciones sanitarias que deben implementarse o consolidarse en el Sector Salud. Estas intervenciones demandarán un mayor presupuesto del Estado para lograr equipamiento, infraestructura y talento humano calificado; y mayor protagonismo de todos los empleados públicos, desde el presidente de la República, ministros, el personal asistencial, administrativo y agentes comunitarios voluntarios; apoyados por las organizaciones civiles, logrando poner a la TB en la agenda pública, como ahora lo es la lucha contra la anemia.

En prevención. Implementar esquemas de tratamiento acortados de la TB latente basados en rifapentina e isoniacida de 12 dosis semanales (régimen $3 \mathrm{HP}$ ) para mejorar la adherencia al tratamiento, con una eficacia similar al régimen de dosis diarias de isoniacida durante 9 meses ${ }^{[4]}$.

En diagnóstico. Expandir el uso de la baciloscopía con fluorescencia por microscopía LED, implementar a nivel nacional la prueba molecular Xpert MTB/Rif ${ }^{\oplus}$ en su versión ultra, para muestras pulmonares y extrapulmonares, reemplazando a la baciloscopía en zonas donde la tasa de incidencia sea mayor a 100 casos por 100 mil habitantes y una prevalencia de más de $10 \%$ de TB MDR [5]. Descentralizar el cultivo y prueba de sensibilidad en sistema BACTEC en medio líquido MGIT. Expandir la tipificación de micobacterias y la detección de resistencia a isoniacida, rifampicina, quinolonas e inyectables de segunda línea por el método Genotype ${ }^{\circledR}$ MTBDR plus y Genotype ${ }^{\circledR}$ MTBDR second line en los laboratorios 
regionales o macroregionales del país. Implementar una real red de radiología digital con lectura a distancia de placas de tórax que sirva de tamizaje de TB activa, como se hacía con las fotos Röntgen y lograr un mayor acceso a tomografía espiral multicorte (TEM) en todas las ciudades capitales del país ${ }^{[6]}$.

En tratamiento de la TB sensible. Implementar el tratamiento diario, en primera y segunda fase, del régimen RHZE (rifampicina, isoniazida, pirazinamida, etambutol) de lunes a domingo, consolidando la presentación en dosis fijas combinadas y la administración del tratamiento directamente observado (TDO) en el domicilio del paciente por agentes comunitarios o familiares, con evaluaciones del paciente cada siete o 15 días en el establecimiento de salud [7], dejando como última opción el TDO basado en el establecimiento de salud, según lo propuesto por la OMS [8], dada su nula contribución en el resultado clínico y el mayor riesgo de contagio entre pacientes ${ }^{[9]}$.

En tratamiento de la TB resistente. Implementar el esquema acortado de 9 meses para la TB MDR según recomendaciones de la OMS. Implementar esquemas para la TB pre-XDR y TB XDR incorporando las recomendaciones de la OMS del 2018 que prioriza el uso de fluoroquinolonas, linezolid, bedaquilina, clofazimina, delamanid. Expandir el TDO centrado en el paciente, que combina un modelo de atención hospitalaria y en el domicilio por personal de enfermería altamente capacitado en la administración de estos medicamentos ${ }^{[10]}$.

Implementar centros especializados en atención exclusiva de pacientes con formas graves de TB y con frotis de esputo positivo. Que cuenten con sala de emergencia, unidad de cuidados intensivos (UCI), sala de operaciones, sala de recuperación y salas de hospitalización. Todas con un adecuado sistema de control de infecciones (ventilación natural y mecánica apropiada) y bioseguridad. En la mayoría de hospitales del país a los pacientes con insuficiencia respiratoria por TB no son admitidos en las UCls, por el riesgo de desencadenar un brote nosocomial.

En tratamiento centrado en el paciente para casos de alto riesgo de pérdida de seguimiento. Crear servicios de tuberculosis en los hospitales de salud mental de las capitales de región donde puedan manejarse personas con TB y adicción a drogas, alcoholismo, psicosis, conducta anti social, entre otros; que cuenten con psiquiatras, infectólogos, neumólogos e internistas con las competencias suficientes para manejar a estas personas de manera institucionalizada hasta que sean declaradas como curadas. Un hospital que se presta para esto a corto plazo, por su extensión de terreno, es el Hospital Larco Herrera en Magdalena del Mar. Asimismo, fortalecer y replicar la experiencia de sanatorios modernos, como el Hogar San Pedro, en Huarochirí - Lima, que alberguen a pacientes de provincia, pobres extremos y personas de calle, mientras reciben su tratamiento.

Mientras se implementan estas medidas, se debe impulsar la investigación en TB, promoviendo la realización de ensayos clínicos multicéntricos, así como a la investigación operativa sobre el actual programa de control de TB e investigación en ciencias básicas.

Finalmente, invocamos a las autoridades nacionales el financiamiento, con tesoro público y alianzas público privadas, de las intervenciones sanitarias propuestas y otorguen facilidades para su implementación por el MINSA, la Seguridad Social, el Instituto Penitenciario y las Sanidades de las Fuerzas Armadas y Policiales. Asimismo, el llamado a las universidades para que prioricen la investigación en TB en sus alumnos de pre y post grado. Por último, un llamado a los colegas médicos que son líderes de los servicios de salud en todo nivel de atención; tienen la obligación de ser expertos en el control y prevención de la TB y guiar a todo su equipo hacia el cumplimiento de la Estrategia Fin de la Tuberculosis al 2035. La siguiente generación nos lo va a agradecer.

\section{REFERENCIAS BIBLIOGRÁFICAS}

1. World Health Organization. Global Tuberculosis Report 2018. Geneva: WHO; 2018.

2. Alarcón V, Alarcón E, Figueroa C, Mendoza-Ticona A. Tuberculosis en el Perú: Situación epidemiológica, avances y desafíos para su control. Rev Peru Med Exp Salud Publica. 2017;34(2):299-310.

3. World Health Organization. The End of TB Strategy [Internet]. Geneva: WHO; 2015 [citado el 8 de noviembre de 2018]. Disponible en: http://www.who.int/tb/End_TB_brochure.pdf

4. Sterling TR, Villarino ME, Borisov AS, Shang N, Gordin F, BlivenSizemore $E$, et al. Three months of rifapentine and isoniazid for latent tuberculosis infection. N Engl J Med. 2011 Dec 8;365(23):2155-66.

5. World Health Organization. Using the Xpert MTB/RIF assay to detect pulmonary and extrapulmonary tuberculosis and rifampicin resistance in adults and children. Geneva: WHO; 2013.
6. World Health Organization. Chest radiography in tuberculosis detection: summary of current WHO recommendations and guidance on programmatic approaches. Geneva; WHO; 2016.

7. Gallardo CR, Rigau Comas D, Valderrama Rodríguez A, Roqué i Figuls $M$, Parker LA, et al. Fixed-dose combinations of drugs versus singledrug formulations for treating pulmonary tuberculosis. Cochrane Database Syst Rev. 2016;(5):CD009913.

8. World Health Organization. Guidelines for treatment of drugsusceptible tuberculosis and patient care. 2017 Update. Geneve: WHO; 2017.

9. Karumbi J, Garner P. Directly observed therapy for treating tuberculosis. Cochrane Database Syst Rev. 2015(5):CD003343.

10. World Health Organization. WHO Treatment guidelines for drugresistant tuberculosis - 2016 update. Geneva: WHO; 2016. 